General Mathematics Vol. 27, No. 1 (2019), 3-15

DOI:10.2478/gm-2019-0001

\title{
sciendo
}

\section{On Neighbor chromatic number of grid and torus graphs ${ }^{1}$}

\author{
B. Chaluvaraju, C. Appajigowda
}

\begin{abstract}
A set $S \subseteq V$ is a neighborhood set of a graph $G=(V, E)$, if $G=\bigcup_{v \in S}\langle N[v]\rangle$, where $\langle N[v]\rangle$ is the subgraph of a graph $G$ induced by $v$ and all vertices adjacent to $v$. A neighborhood set $S$ is said to be a neighbor coloring set if it contains at least one vertex from each color class of a graph $G$, where color class of a colored graph is the set of vertices having one particular color. The neighbor chromatic number $\chi_{\eta}(G)$ is the minimum cardinality of a neighbor coloring set of a graph $G$. In this article, some results on neighbor chromatic number of Cartesian products of two paths (grid graph) and cycles (torus graphs) are explored.
\end{abstract}

2010 Mathematics Subject Classification: 05C15, 05C70.

Key words and phrases: Neighborhood number, chromatic number, neighbor chromatic number, grid graph, torus graph.

\section{Introduction}

All graphs considered here are finite, undirected and connected with no loops and multiple edges. As usual $p=|V|$ and $q=|E|$ denote the number of vertices and edges of a graph $G$, respectively. In general, we use $\langle X\rangle$ to denote the sub graph induced by the set of vertices $X . N(v)$ and $N[v]$ denote the open and closed neighborhoods of a vertex $v$, respectively. For graph-theoretical terminology and notation not defined here we follow [4].

According to Hammack et al. [14, the Cartesian products of graphs were defined in 1912 by Whitehead and Russel. They were repeatedly rediscovered later, notably by Sabidussi (1960) and independently by Vizing (1963). Note that $K_{2} \square K_{2}=C_{4}$, that is, the Cartesian products of two edges is a square. This is the motivation for

\footnotetext{
${ }^{1}$ Received 25 April, 2018

Accepted for publication (in revised form) 17 June, 2018
} 
the introduction of the notation $\square$ for the Cartesian product (or Box product). The Cartesian product of two graphs $G$ and $H$, denoted by $G \square H$, is a graph with a vertex set $V(G \square H)=V(G) \times V(H)$, that is, the set $\{(g, h): g \in G, h \in H\}$. The edge set of $G \square H$ consists of all pairs $\left[\left(g_{1}, h_{1}\right),\left(g_{2}, h_{2}\right)\right]$ of vertices with $\left[g_{1}, g_{2}\right] \in E(G)$ and $h_{1}=h_{2}$, or $g_{1}=g_{2}$ and $\left[h_{1}, h_{2}\right] \in E(H)$. For more details, see [6] and [7].

A coloring of a graph is an assignment of colors to its vertices so that no two adjacent vertices have the same color. A color class of a colored graph is the set of vertices having one particular color. The minimum number of colors that can be used to color the vertices is called a chromatic number and is denoted by $\chi(G)$. A set $S \subseteq V(G)$ is a neighborhood set of $G$, if $G=\bigcup_{v \in S}\langle N[v]\rangle$, where $\langle N[v]\rangle$ is the subgraph of a graph $G$ induced by $v$ and all vertices adjacent to $v$. The neighborhood number $\eta(G)$ is the minimum cardinality of a neighborhood set of $G$. This concept was first introduced by Sampathkumar and Neeralagi [16] and studied in [1] and [12. A neighborhood set $S$ with minimum cardinality is called $\eta$ - set of $G$.

Analogously, the concept of a neighbor chromatic number was introduced by Chaluvaraju et al. in [2] as follows:

Let $G$ be a colored graph. A neighborhood set $S$ of $G$ is said to be a neighbor coloring set if it contains at least one vertex from each color class of $G$. The minimum cardinality taken over all neighbor coloring sets for all proper colorings of $G$ is called neighbor chromatic number and is denoted by $\chi_{\eta}(G)$. For more details on related concepts, we refer the reader to [9], [11], [13] and [17].

The main goal of this paper is to obtain some results about the neighbor chromatic numbers of certain classes of Cartesian products of graphs.

\section{$2 \quad$ Existing Results}

In 1957 Sabidussi proved in [15] the following result about the chromatic number of the Cartesian product of two graphs. Since then this result has been rediscovered several times.

Proposition 1 For any two graphs $G$ and $H$,

$$
\chi(G \square H)=\max \{\chi(G), \chi(H)\} .
$$

The possible values of neighbor chromatic numbers of complete graphs, paths and cycles are obtained in [2] and are given in the following propositions.

Proposition 2 For any complete graph $K_{p}$ with $p \geq 2$ vertices,

$$
\chi_{\eta}\left(K_{p}\right)=p .
$$

Proposition 3 For any path $P_{p}$ with $p \geq 2$ vertices,

$$
\chi_{\eta}\left(P_{p}\right)= \begin{cases}2, & \text { if } p=2 \\ \left\lceil\frac{p}{2}\right\rceil, & \text { if } p>2 .\end{cases}
$$


Proposition 4 For any cycle $C_{p}$ with $p \geq 3$ vertices,

$$
\chi_{\eta}\left(C_{p}\right)= \begin{cases}\frac{p}{2}+1, & \text { if } p=2 r ; r \geq 2 \\ \left\lceil\frac{p}{2}\right\rceil, & \text { if } p=2 r+1 ; r \geq 2 .\end{cases}
$$

Observation 1 In a graph $G$ with $\chi$-coloring, not all neighborhood sets are neighbor coloring sets.

For example, consider a complete graph $K_{p}$ with $p \geq 2$ vertices and $V\left(K_{p}\right)=$ $\left\{v_{1}, v_{2}, \ldots, v_{p}\right\}$. The neighborhood set is $v_{1}$, which is not a neighbor coloring set. The set of all vertices of $V\left(K_{p}\right)$ is the neighbor coloring set. Similarly, many more graphs can be expected.

\section{The Grid graph}

The $m \square n$ grid graph is a Cartesian products $P_{m} \square P_{n}$ of a path of length $m-1$ and a path of length $n-1$. The first results on the domination number of grid was obtained by Jacobson and Kinch [8] and studied in [3].

Observation 2 For any two paths $P_{m}$ and $P_{n}$ with $m, n \geq 2$,

(i) $\chi\left(P_{m} \square P_{n}\right) \leq \chi_{\eta}\left(P_{m} \square P_{n}\right)$,

(ii) $\eta\left(P_{m} \square P_{n}\right) \leq \chi_{\eta}\left(P_{m} \square P_{n}\right)$.

For example, consider a grid graph $P_{2} \square P_{2}$, we have $\chi\left(P_{2} \square P_{2}\right)=2=\eta\left(P_{2} \square P_{2}\right)$ and $\chi_{\eta}\left(P_{2} \square P_{2}\right)=3$.

Theorem 1 For any two paths $P_{m}$ and $P_{n}$,

$$
\chi_{\eta}\left(P_{m} \square P_{n}\right)= \begin{cases}\frac{m n-1}{2}, & \text { if } m \text { and } n \text { are odd } \\ \frac{m n}{2}+1, & \text { otherwise. }\end{cases}
$$

Proof. Let the vertices of a path $P_{m}$ be labeled $u_{1}, u_{2}, \ldots, u_{m}$ and the vertices of a path $P_{n}$ be labeled $v_{1}, v_{2}, \ldots, v_{n}$. We know that, any grid graph is a bipartite graph, hence, $\chi\left(P_{m} \square P_{n}\right)=2$. Then the following cases arise:

Case 1.m $_{m+1}$ and $n$ are odd. Then the set $A=\bigcup_{k=1}^{\frac{m+1}{2}}\left\{\left(u_{2 k-1}, v_{2 t}\right): 1 \leq t \leq \frac{n-1}{2}\right\} \cup \bigcup_{k=1}^{\frac{m-1}{2}}\left\{\left(u_{2 k}, v_{2 t-1}\right): 1 \leq t \leq \frac{n+1}{2}\right\}$ is the unique $\eta$ - set which contains vertices of the same color. Hence, $B=\bar{A} \cup\left\{\left(u_{1}, v_{1}\right)\right\}$ is a $\eta$ - set which contains both colors. Thus,

$$
\begin{aligned}
\chi_{\eta}\left(P_{m} \square P_{n}\right) & =\left(\frac{m+1}{2}\right)\left(\frac{n-1}{2}\right)+\left(\frac{m-1}{2}\right)\left(\frac{n+1}{2}\right) \\
& =\frac{(m+1)(n-1)+(m-1)(n+1)}{4} \\
& =\frac{m n-1}{2} .
\end{aligned}
$$


Case $\mathscr{2}_{m} m$ and $n$ are even. Then the sets $A=\bigcup_{k=1}^{\frac{m}{2}}:\left\{\left(u_{2 k-1}, v_{2 t}\right): 1 \leq t \leq \frac{n}{2}\right\} \cup \bigcup_{k=1}^{\frac{m}{2}}:\left\{\left(u_{2 k}, v_{2 t-1}\right): 1 \leq t \leq \frac{n}{2}\right\}$ and $B=\bigcup_{k=1}^{\frac{m}{2}}\left\{\left(u_{k}, v_{2 t-1}\right): 1 \leq t \leq \frac{n}{2}\right\} \cup \bigcup_{k=1}^{\frac{m}{2}}\left\{\left(u_{k}, v_{2 t}\right): 1 \leq t \leq \frac{n}{2}\right\}$ are the only two $\eta$ - sets, each containing vertices of the same color. Hence, $A \cup\left\{\left(u_{1}, v_{1}\right)\right\}$ or $B \cup\left\{\left(u_{2}, v_{1}\right)\right\}$ is a $\chi_{\eta}$ - set, which contains both colors. Thus,

$$
\begin{aligned}
\chi_{\eta}\left(P_{m} \square P_{n}\right) & =\frac{m}{2}\left(\frac{n}{2}\right)+\frac{m}{2}\left(\frac{n}{2}\right)+1 \\
& =2\left(\frac{m n}{4}\right)+1 \\
& =\left(\frac{m n}{2}\right)+1 .
\end{aligned}
$$

Case $3_{\dot{m}} m$ is even and $n$ is odd. Then the sets

$A=\bigcup_{k=1}^{\frac{m}{2}}\left\{\left(u_{2 k-1}, v_{2 t-1}\right): 1 \leq t \leq \frac{n+1}{2}\right\} \cup \bigcup_{m=1}^{\frac{m}{2}}\left\{\left(u_{2 k}, v_{2 t}\right): 1 \leq t \leq \frac{n-1}{2}\right\}$ and $B=\bigcup_{k=1}^{\frac{m}{2}}\left\{\left(u_{2 k-1}, v_{2 t}\right): 1 \leq t \leq \frac{n-1}{2}\right\} \cup \bigcup_{k=1}^{\frac{m}{2}}\left\{\left(u_{2 k}, v_{2 t-1}\right): 1 \leq t \leq \frac{n+1}{2}\right\}$ are the only two $\eta$ - sets, each containing the same color. Hence, $A \cup\left\{\left(u_{2}, v_{1}\right)\right\}$ or $B \cup\left\{\left(u_{1}, v_{1}\right)\right\}$ is a $\chi_{\eta}$ - set. Thus,

$$
\begin{aligned}
\chi_{\eta}\left(P_{m} \square P_{n}\right) & =\frac{m}{2}\left(\frac{n+1}{2}\right)+\frac{m}{2}\left(\frac{n-1}{2}\right)+1 \\
& =2\left(\frac{m n}{4}\right)+1 \\
& =\left(\frac{m n}{2}\right)+1 .
\end{aligned}
$$

Case 4. $m$ is odd and $n$ is even. This case is equivalent to Case 3.

\section{The Torus graph}

The torus graph $C_{m} \square C_{n}$ is the Cartesian product of a cycle of length $m$ and a cycle of length $n$ with $m, n \geq 3$ vertices. For more detail, we refer the reader to [10].

Theorem 2 For any two cycles with $m=3$ and $n \geq 3$ vertices,

$$
\chi_{\eta}\left(C_{3} \square C_{n}\right)= \begin{cases}5, & \text { if } n=3 \\ \frac{3 n}{2}, & \text { if } n \text { is even } \\ \frac{3(n+1)}{2}, & \text { if } n>3 \text { is odd. }\end{cases}
$$

Proof. Let the vertices of the cycle $C_{3}$ be labeled $u_{1}, u_{2}, u_{3}$ and the vertices of the cycle $C_{n}$ labeled $v_{1}, v_{2}, \ldots, v_{n}$. Then the following cases arise.

Case 1. $n=3$.

In this case, the unique (upto isomorphism) coloring of graph $C_{3} \square C_{3}$ with 3 colors is shown in Figure - 1 . 


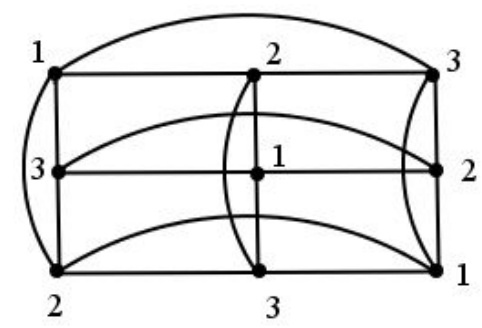

Figure 1: Chromatic number of $C_{3} \square C_{3}$

In $C_{3} \square C_{3}$, the set $A=\left\{\left(u_{2}, v_{1}\right),\left(u_{1}, v_{2}\right),\left(u_{3}, v_{2}\right),\left(u_{2}, v_{3}\right)\right\}$ is an unique $\eta$ - set. The set $A$ (and every other $\eta$-set) includes only two colors. Hence, $B=A \cup\left\{\left(u_{2}, v_{2}\right)\right\}$ is a $\chi_{\eta}$ - set. Therefore, $\chi_{\eta}\left(C_{3} \square C_{3}\right)=5$.

Case 2. $n$ is even.

Since the graph $C_{3} \square C_{n}$ contains at least one graph $C_{3}, \chi\left(C_{3} \square C_{n}\right) \geq 3$. Now we prove $\chi\left(C_{3} \square C_{n}\right)=3$ by showing the existence of a 3-coloring.

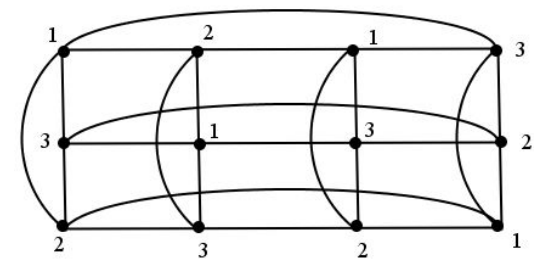

Figure 2: Chromatic number of $C_{3} \square C_{4}$

We begin with the graph $C_{3} \square C_{4}$ colored as shown in Figure - 2, and at each step we add two consecutive columns, say column $j$ and column $j+1$, after column three and before column four, and we color the vertices of column $j$ as the vertices of column two and we color the vertices of $j+1$ as the vertices of column three, as shown in Figure - 3 .

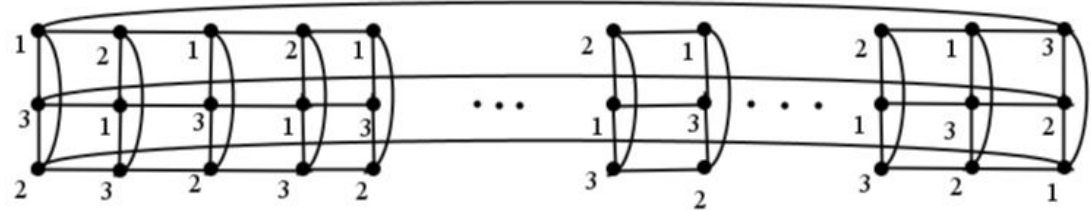

Figure 3: Chromatic number of $C_{3} \square C_{n}$

We continue this process of adding consecutive columns untill we have n columns. Since we have not used new color in the process, $\chi\left(C_{3} \square C_{n}\right)=3$. But $\eta\left(C_{3}\right)=$ 
$\chi\left(C_{3}\right)=3$.

Obviously, the $C_{3} \square C_{n}$ contain the total number of vertices are multiples of 3 . Thus, the coloring starts with minimum 3 colors, which implies the set

$A=\left\{\left(u_{1}, v_{2 t}\right): 1 \leq t \leq \frac{n}{2}\right\} \cup\left\{\left(u_{2}, v_{2 t-1}\right): 1 \leq t \leq \frac{n}{2}\right\} \cup\left\{\left(u_{3}, v_{2 t}\right): 1 \leq t \leq \frac{n}{2}\right\}$ is a $\eta$ - set containing $\left(u_{1}, v_{2}\right),\left(u_{1}, v_{n}\right)$ and $\left(u_{3}, v_{n}\right)$ with colors 2, 3 and 1 respectively. Hence, $\chi_{\eta}\left(C_{3} \square C_{n}\right)=\frac{n}{2}+\frac{n}{2}+\frac{n}{2}=\frac{3 n}{2}$.

Case 3. $n \geq 5$ is odd.

Since the graph $C_{3} \square C_{n}$ contains at least one graph $C_{3}, \chi\left(C_{3} \square C_{n}\right) \geq 3$. Now we prove $\chi\left(C_{3} \square C_{n}\right)=3$ by showing the existence of a 3-coloring.

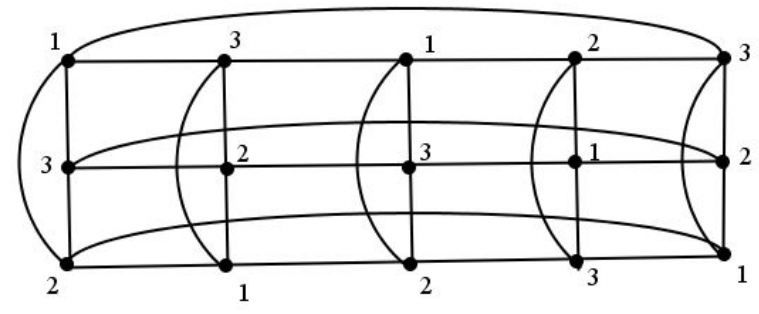

Figure 4: Chromatic number of $C_{3} \square C_{5}$

We begin with the graph $C_{3} \square C_{5}$ colored as shown in Figure - 4, and at each step we add two consecutive columns, say column $j$ and column $j+1$, after column four and before column five and we color the vertices of column $j$ as the vertices of column three and we color the vertices of column $j+1$ as the vertices of column four, as shown in Figure - 5 .

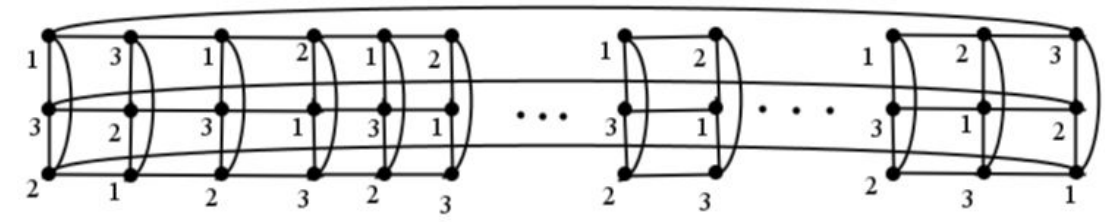

Figure 5: Chromatic number of $C_{3} \square C_{n} ; n$ is odd

We continue this process of adding consecutive columns untill we have $n$ columns. Since we have not used new colors in the process, $\chi\left(C_{3} \square C_{n}\right)=3$. Since $\eta\left(C_{3}\right)=$ $\chi\left(C_{3}\right)=3$. So that $C_{3} \square C_{n}$ where $n \geq 5$ (odd) vertices contain both odd cycles and the total number of vertices are multiples of 3 . Thus the coloring starts with minimum 3 colors, which implies the set $A=\left\{\left(u_{1}, v_{2 t}\right): 1 \leq t \leq \frac{n-1}{2}\right\} \cup\left\{\left(u_{2}, v_{2 t-1}\right): 1 \leq\right.$ $\left.t \leq \frac{n+1}{2}\right\} \cup\left\{\left(u_{3}, v_{2 t}\right): 1 \leq t \leq \frac{n-1}{2}\right\} \cup\left\{\left(u_{1}, v_{n}\right),\left(u_{3}, v_{n}\right)\right\}$ is the $\eta$ - set containing $\left(u_{1}, v_{n}\right),\left(u_{2}, v_{n}\right)$ and $\left(u_{3}, v_{n}\right)$ with colors 3, 2 and 1 , respectively. Hence, $\chi_{\eta}\left(C_{3} \square C_{n}\right)=\frac{n-1}{2}+\frac{n+1}{2}+\frac{n-1}{2}+2=\frac{3(n+1)}{2}$. 
Theorem 3 For any two cycles with $m, n \geq 4$ vertices,

$$
\chi_{\eta}\left(C_{m} \square C_{n}\right)= \begin{cases}\frac{m n}{2}+1, & \text { if both } m \text { and } n \text { are even } \\ \frac{n(m-1)}{2}+n, & \text { if } m \text { is odd and } n \text { is even } \\ \frac{m(n-1)}{2}+m, & \text { if } m \text { is even and } n \text { is odd } \\ \frac{(m-1)(n-1)}{2}+(m+n)-2, & \text { if both } m \text { and } n \text { are odd. }\end{cases}
$$

Proof. Let the vertices of a cycle $C_{m}$ be labeled $u_{1}, u_{2}, \ldots, u_{m}$ and the vertices of a cycle $C_{n}$ be labeled $v_{1}, v_{2}, \ldots, v_{n}$. The set $\left\{\left(u_{i}, v_{j}\right): u_{i} \in V\left(C_{m}\right)\right.$ and $\left.v_{j} \in V\left(C_{n}\right)\right\}$ is the vertex set of $C_{m} \square C_{n}$. Then the following cases arise:

Case 1. $m$ and $n$ are even.

First we prove $\chi\left(C_{m} \square C_{n}\right)=2$. Since both $m$ and $n$ are even, the graph $C_{m} \square C_{n}$ contains no odd cycle. Hence, $C_{m} \square C_{n}$ is a bipartite graph containing at least one edge. Therefore, $\chi\left(C_{m} \square C_{n}\right)=2$, with $\chi$-coloring defined as follows:

Color the vertices of the first row and the vertices of every other row with colors $1,2,1,2, \cdots$ and color the vertices of all remaining rows with colors $2,1,2,1, \cdots$. In this way, we get a 2-coloring of $C_{m} \square C_{n}$ as shown in Figure - 6 .

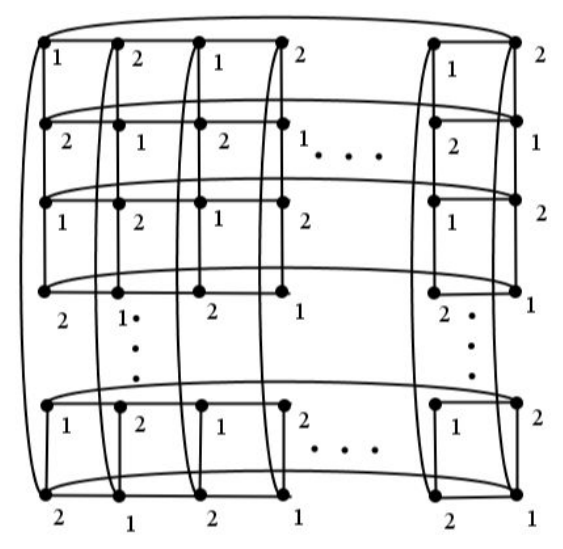

Figure 6: Chromatic number of $C_{m} \square C_{n} ; m$ and $n$ is even

Let $A=\bigcup_{k}^{\frac{n}{2}}\left\{\left(u_{2 t}, v_{2 k-1}\right): 1 \leq t \leq \frac{m}{2}\right\} \cup \bigcup_{k}^{\frac{n}{2}}\left\{\left(u_{2 t-1}, v_{2 k}\right): 1 \leq t \leq \frac{m}{2}\right\}$ and $B=\bigcup_{k}^{\frac{n}{2}}\left\{\left(u_{2 t-1}, v_{2 k-1}\right): 1 \leq t \leq \frac{m}{2}\right\} \cup \bigcup_{k}^{\frac{n}{2}}\left\{\left(u_{2 t}, v_{2 k}\right): 1 \leq t \leq \frac{m}{2}\right\}$ are the only two $\eta$ - sets of $C_{m} \square C_{n}$ but each of the sets $A$ and $B$ contains vertices of the same color. Therefore, $A \cup\left\{\left(u_{1}, v_{1}\right)\right\}$ is a $\chi_{\eta}$ - set of $C_{m} \square C_{n}$. Hence, $\chi_{\eta}\left(C_{m} \square C_{n}\right)=$ $\frac{m}{2}\left(\frac{n}{2}\right)+\frac{m}{2}\left(\frac{n}{2}\right)+1=\frac{m n}{2}+1$.

Case 2. $m$ is odd and $n$ is even.

Since $m$ is odd, the graph $C_{m} \square C_{n}$ contains an odd cycle, therefore $\chi\left(C_{m} \square C_{n}\right) \geq 3$. 
Now we prove $\chi\left(C_{m} \square C_{n}\right)=3$ by showing the existence of a 3-coloring for $C_{m} \square C_{n}$. Consider $C_{3} \square C_{3}$ with 3-coloring, as shown in Figure 7 .

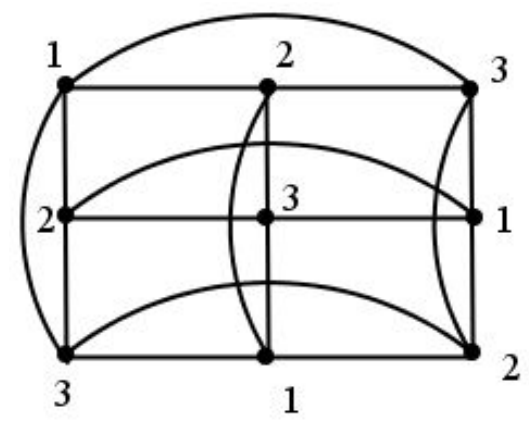

Figure 7: Chromatic number of $C_{3} \square C_{3}$

Since $n \geq 4$ (even), we extend $C_{3} \square C_{3}$ to $C_{3} \square C_{4}$ by adding one extra column of vertices after the second column and before the third column, with colors the same as the colors used for the corresponding vertices in the first column, as shown in Figure 8. Hence we have the following color classes.

(i) $\bigcup_{k}^{\frac{n}{2}}\left\{\left(u_{2 t}, v_{2 k-1}\right): 1 \leq t \leq \frac{m-1}{2}\right\}=\bigcup_{k}^{2}\left\{\left(u_{2 t}, v_{2 k-1}\right): 1 \leq t \leq 1\right\}$.

The color classes $\left(u_{2}, v_{1}\right)$ for $k=1, t=1$ and $\left(u_{2}, v_{3}\right)$ for $k=2, t=1$.

(ii) $\bigcup_{k}^{\frac{n}{2}}\left\{\left(u_{2 t-1}, v_{2 k}\right): 1 \leq t \leq \frac{m-1}{2}\right\}=\bigcup_{k}^{2}\left\{\left(u_{2 t-1}, v_{2 k}\right): 1 \leq t \leq 1\right\}$.

The color classes $\left(u_{1}, v_{2}\right)$ for $k=1, t=1$ and $\left(u_{1}, v_{4}\right)$ for $k=2, t=1$.

(iii) $\left\{\left(u_{m}, v_{j}\right): 1 \leq j \leq n\right\}=\left\{\left(u_{m}, v_{j}\right): 1 \leq j \leq 4\right\}$.

The color classes $\left(u_{3}, v_{1}\right)$ for $m=3, n=1 ;\left(u_{3}, v_{2}\right)$ for $m=3$ and $n=2$;

$\left(u_{3}, v_{3}\right)$ for $m=3, n=3$ and $\left(u_{3}, v_{4}\right)$ for $m=3, n=4$.

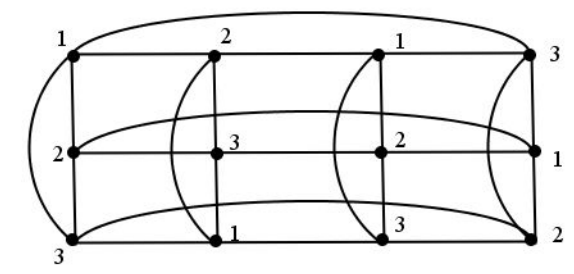

Figure 8: Chromatic number of $C_{3} \square C_{4}$

From (i), (ii) and (iii), we have the $\eta$-set of $C_{3} \square C_{4}$ as follows:

$$
A=\left\{\left(u_{2}, v_{1}\right),\left(u_{2}, v_{3}\right),\left(u_{1}, v_{2}\right),\left(u_{1}, v_{4}\right),\left(u_{3}, v_{1}\right),\left(u_{3}, v_{2}\right),\left(u_{3}, v_{3}\right),\left(u_{3}, v_{4}\right)\right\}
$$

The set $A$ containing at least one vertex from each color class as follows: 
(i) $V_{1}$ containing the color class 1.

That is, $V_{1}=\left\{\left(u_{1}, v_{1}\right),\left(u_{1}, v_{2}\right),\left(u_{2}, v_{4}\right),\left(u_{3}, v_{2}\right)\right\}$.

(ii) $V_{2}$ containing the color class 2.

That is, $V_{2}=\left\{\left(u_{1}, v_{2}\right),\left(u_{2}, v_{1}\right),\left(u_{2}, v_{3}\right),\left(u_{3}, v_{4}\right)\right\}$.

(iii) $V_{3}$ containing the color class 3.

That is, $V_{3}=\left\{\left(u_{1}, v_{4}\right),\left(u_{2}, v_{2}\right),\left(u_{3}, v_{1}\right),\left(u_{3}, v_{3}\right)\right\}$.

By virtue of the above fact that the set $A$ is a $\eta$-set as well as $\chi_{\eta}$-set of $C_{3} \square C_{4}$.

Now we extend $C_{3} \square C_{4}$ to $C_{3} \square C_{n}$ by retaining the 3-coloring of $C_{3} \square C_{4}$, at each step adding 2-columns of vertices, say column $j$ and column $j+1$, after the third column and before the fourth column and we color the vertices of column $j$ as the vertices of column two and we color the vertices of column $j+1$ as the vertices of column one, as shown in Figure - 9. We continue this process untill we have n-

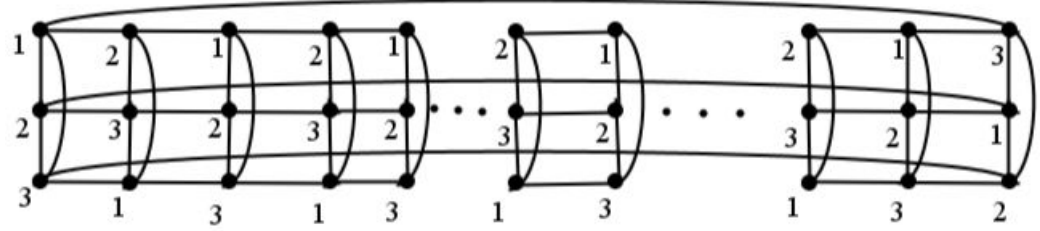

Figure 9: Chromatic number of $C_{3} \square C_{n} ; n$ is even

columns in total. Since we have not used new colors in the process, the graph $C_{3} \square C_{n}$ has a 3-coloring. Now we extend $C_{3} \square C_{n}$ to $C_{m} \square C_{n}$ by retaining the 3-coloring.

We begin with the graph $C_{3} \square C_{n}$ colored as shown in Figure - 9, and at each step we add two consecutive rows of vertices, say row $i$ and row $i+1$, after the second row and before the third row and we color the vertices of row $i$ as the vertices of the first row and we color the vertices of row $i+1$ as the vertices of the second row, as shown in Figure 10.

We continue this process untill we have $m$-rows in total. Since we have not used new colors in the process, the graph $C_{m} \square C_{n}$ has a 3-coloring. Hence, $\chi\left(C_{m} \square C_{n}\right)=$ 3. Since $\eta\left(C_{3}\right)=\chi\left(C_{3}\right)=3 ; C_{m} \square C_{n}$ where $m$ is odd and $n$ is even number vertices contain the total number of vertices are multiples of 3 . Thus the coloring starts with minimum 3 colors, which implies the set $A=\bigcup_{k}^{\frac{n}{2}}\left\{\left(u_{2 t}, v_{2 k-1}\right): 1 \leq\right.$ $t \leq \frac{m-1}{2} \cup \bigcup_{k}^{\frac{n}{2}}\left\{\left(u_{2 t-1}, v_{2 k}\right): 1 \leq t \leq \frac{m-1}{2}\right\} \cup\left\{\left(u_{m}, v_{j}\right): 1 \leq j \leq n\right\}$ is a $\eta$ - set containing at least one vertex from each color class. By virtue of the fact that, the $A$ is a $\eta$-set as well as $\chi_{\eta}$-set of $C_{3} \square C_{n}$ and $A$ is a minimum $\eta$-set. Hence,

$$
\chi_{\eta}\left(C_{m} \square C_{n}\right)=\frac{n}{2}\left(\frac{m-1}{2}\right)+\frac{n}{2}\left(\frac{m-1}{2}\right)+n=\frac{n(m-1)}{2}+n .
$$

Case 3. $m$ is even and $n$ is odd. This case is equivalent to Case 2.

Case 4. $m$ and $n$ are odd.

Since both $m$ and $n$ are odd, the graph $C_{m} \square C_{n}$ contains atleast one odd cycle, 


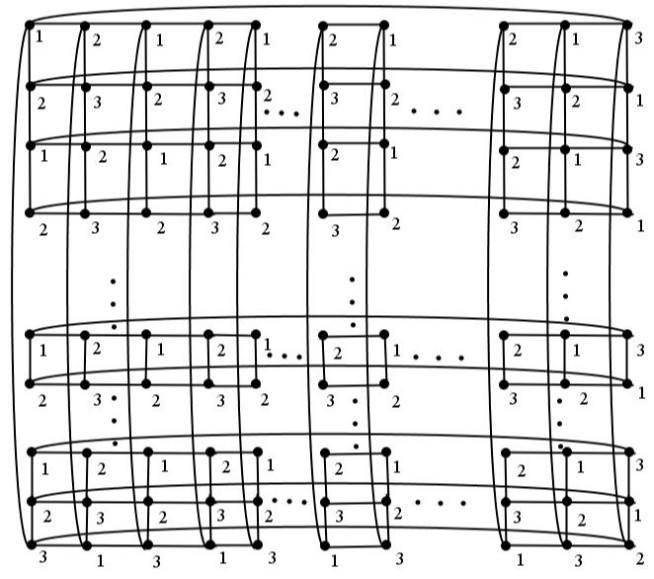

Figure 10: Chromatic number of $C_{m} \square C_{n} ; m$ is odd and $n$ is even

therefore $\chi\left(C_{m} \square C_{n}\right) \geq 3$. Now we prove $\chi\left(C_{m} \square C_{n}\right)=3$ by showing the existence of a 3-coloring for $C_{m} \square C_{n}$.

Consider $C_{3} \square C_{3}$ with 3-coloring, as shown in Figure - 7. Hence we have the following color classes.

(i) $\bigcup_{k}^{\frac{n-1}{2}}\left\{\left(u_{2 t}, v_{2 k-1}\right): 1 \leq t \leq \frac{m-1}{2}\right\}$.

The color class $\left(u_{2}, v_{1}\right)$ for $k=1, t=1$.

(ii) $\bigcup_{k}^{\frac{n-1}{2}}\left\{\left(u_{2 t-1}, v_{2 k}\right): 1 \leq t \leq \frac{m-1}{2}\right\}$.

The color class $\left(u_{1}, v_{2}\right)$ for $k=1, t=1$.

(iii) $\left\{\left(u_{m}, v_{j}\right): 1 \leq j \leq n-1\right\}$.

The color classes $\left(u_{3}, v_{1}\right)$ for $m=3, i=1$ and $\left(u_{3}, v_{2}\right)$ for $m=3, i=2$.

(iv) $\left\{\left(u_{i}, v_{n}\right): 1 \leq i \leq m-1\right\}$.

The color classes $\left(u_{1}, v_{3}\right)$ for $n=3, i=1$ and $\left(u_{2}, v_{3}\right)$ for $n=3, i=2$.

From (i) - (iv), we have the $\eta$-set of $C_{3} \square C_{3}$ as follows:

$$
A=\left\{\left(u_{2}, v_{1}\right),\left(u_{1}, v_{2}\right),\left(u_{3}, v_{1}\right),\left(u_{3}, v_{2}\right),\left(u_{1}, v_{3}\right),\left(u_{2}, v_{3}\right)\right\} .
$$

The set A containing at least one vertex from each color classes as follows:

(i) $V_{1}$ containing the color class 1 .

That is, $V_{1}=\left\{\left(u_{1}, v_{1}\right),\left(u_{2}, v_{3}\right),\left(u_{3}, v_{2}\right)\right\}$.

(ii) $V_{2}$ containing the color class 2.

That is, $V_{2}=\left\{\left(u_{1}, v_{2}\right),\left(u_{2}, v_{1}\right),\left(u_{3}, v_{3}\right)\right\}$.

(iii) $V_{3}$ containing the color class 3.

That is, $V_{3}=\left\{\left(u_{1}, v_{3}\right),\left(u_{2}, v_{2}\right),\left(u_{3}, v_{1}\right)\right\}$. 
By virtue of the above fact that the set $A$ is a $\eta$-set as well as $\chi_{\eta}$-set of $C_{3} \square C_{3}$.

Now we extend $C_{3} \square C_{3}$ to $C_{3} \square C_{n}$ by retaining the 3-coloring. We begin with the graph $C_{3} \square C_{3}$ colored as shown in Figure 7, and at each step we add two columns of vertices, say column $j$ and column $j+1$, after the second column and before the third column and we color the vertices of column $j$ as the vertices of column one and we color the vertices of column $j+1$ as the vertices of column two, as shown in Figure 11 .

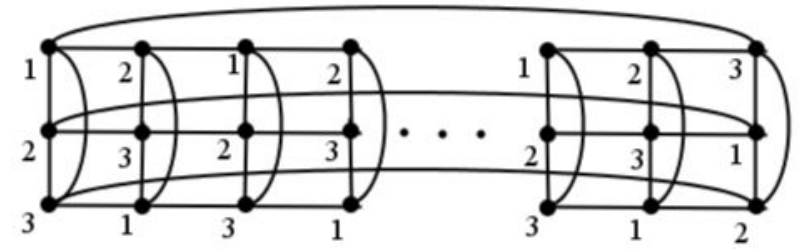

Figure 11: Chromatic number of $C_{3} \square C_{n} ; n$ is odd

We continue this process untill we have $n$-columns in total. Since we have not used any colors in the process, the graph $C_{3} \square C_{n}$ has 3-coloring. Now we extend $C_{3} \square C_{n}$ to $C_{m} \square C_{n}$ by retaining the 3-coloring. We begin with the graph $C_{3} \square C_{n}$ colored as shown in Figure - 11, and each step we add two consecutive rows of vertices, say row $i$ and row $i+1$, after the second row and before the third row and we color the vertices of row $i$ as the vertices of the first row and we color the vertices of row $i+1$ as the vertices of row, as shown in Figure 12.

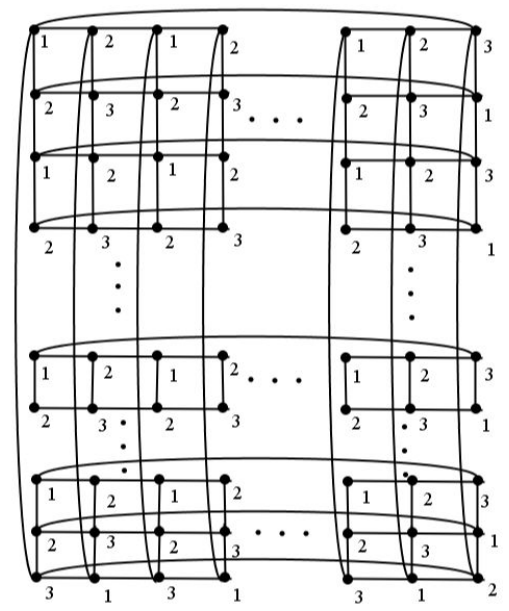

Figure 12: Chromatic number of $C_{m} \square C_{n} ; m$ and $n$ are odd

We continue this process untill we have $m$-rows in total. Since we have not used new colors in the process, the graph $C_{m} \square C_{n}$ has 3-coloring. Hence $\chi\left(C_{m} \square C_{n}\right)=3$. 
Since $\eta\left(C_{3}\right)=\chi\left(C_{3}\right)=3$. So that $C_{m} \square C_{n}$ where $m$ and $n$ are both odd vertices, which implies both odd cycles and the total number of vertices are multiples of 3. Thus the coloring starts with minimum 3 colors and the set is

$A=\bigcup_{k}^{\frac{n-1}{2}}\left\{\left(u_{2 t}, v_{2 k-1}\right): 1 \leq t \leq \frac{m-1}{2}\right\} \cup \bigcup_{k}^{\frac{n-1}{2}}\left\{\left(u_{2 t-1}, v_{2 k}\right): 1 \leq t \leq \frac{m-1}{2}\right\} \cup$ $\left\{\left(u_{m}, v_{j}\right): 1 \leq j \leq n-1\right\} \cup\left\{\left(u_{i}, v_{n}\right): 1 \leq i \leq m-1\right\}$ is a $\eta$ - set containing at least one vertex from each color class. By virtue of the above fact that the set $A$ is a $\eta$-set as well as $\chi_{\eta}$-set of $C_{3} \square C_{n}$ and $A$ is a minimum $\eta$-set. Hence,

$$
\begin{aligned}
\chi_{\eta}\left(C_{m} \square C_{n}\right) & =\frac{n-1}{2}\left(\frac{m-1}{2}\right)+\frac{n-1}{2}\left(\frac{m-1}{2}\right)+(n-1)+(m-1) \\
& =\frac{(m-1)(n-1)}{2}+(m+n)-2 .
\end{aligned}
$$

\section{ACKNOWLEDGEMENT}

The authors would like to thank the anonymous reviewers for their valuable comments and suggestions to improve the quality of the paper.

\section{References}

[1] B. Chaluvaraju, Some parameters on neighborhood number of a graph. Electronic Notes of Discrete Mathematics, Elsevier, vol. 33, 2009, 139-146.

[2] B. Chaluvaraju, C. N. Kumar, C. Appajigowda, The neighbor coloring sets in graphs, International J. of Applied Math. and Computation, vol. 4, no. 3, 2012, 307-311.

[3] T. Y. Chang, Domination numbers of grid graphs, Ph.D. Thesis, Department of Mathematics, University of South Florida, 1992.

[4] F. Harary, Graph theory, Addison-Wesley, Reading Mass, 1969.

[5] T. W. Haynes, S. T. Hedetniemi, P. J. Slater, Fundamentals of Domination in Graphs, Marcel Dekkar, Inc., 1997.

[6] W. Imrich, S. Klavzar, Product Graphs; Structure and Recognition, John Wiley and Sons, New York., 2000.

[7] W. Imrich, S. Klavzar, D. F. Rall, Topics in Graph Theory: Graphs and their Cartesian product, A. K. Peters, Ltd., Wellesley, 2008.

[8] M. S. Jacobson, L. F. Kinch, On the domination number of products of a graph; I, Ars Comb., vol. 10, 1983, 33-44.

[9] T. R. Jensen, B. Toft, Graph Coloring Problem, John Wiley \& Sons, Inc, New York, 1995. 
[10] S. Klavzar, N. Seifter, Dominating Cartesian products of cycles, Discrete Applied Mathematics, vol. 59, 1995, 129-136.

[11] R. H. Lamprey, B. H. Barnes, Product graphs and their applications, Modelling and Simulation, Proc. Fifth Annual Pittsburgh Conference, Instrument Society of America, Pittsburgh, PA., vol. 5, 1974, 1119-1123.

[12] V. R. Kulli, S. C. Sigarkanti, Further results on the neighborhood number of graphs. Indian J. Pure Appl. Math, vol. 23, no. 8, 1992, 575-577.

[13] R. Gera, On Dominator colorings in graphs, Graph Theory Notes of New York LII, 2007, 25-30.

[14] R. Hammack, W. Imrich, S. Klavzar, Hand book of product graph. CRC press, Taylor \& Francis group, LLC, 2011.

[15] G. Sabidussi, Graphs with given group and given graph-theoretical properties, Canad. J. Math., vol. 9, 1957, 515-525.

[16] E. Sampathkumar, P. S. Neeralagi, The neighborhood number of a graph, Indian J. Pure and Appl. Math., vol. 16, no. 2, 1985, 126-132.

[17] Y. B. Venkatakrishnan, V. Swaminathan, Colour class domination numbers of some classes of graphs, Algebra and Discrete Mathematics, vol. 18, no. 2, 2014, 301-305.

\section{B. Chaluvaraju}

Bangalore University

Department of Mathematics

Jnana Bharathi Campus

Bangalore-560 056

INDIA

e-mail: bchaluvaraju@gmail.com

\section{Appajigowda}

Bangalore University

Department of Mathematics

Jnana Bharathi Campus

Bangalore-560 056

INDIA

e-mail: appajigowdac@gmail.com 